\title{
Les Hétéroptères aquatiques du Liban. I. Inventaire faunistique.
}

\author{
N. Nieser ${ }^{1}$ \\ Z. Moubayed2
}

Mots clés : Hétéroptères aquatiques, Liban, Région levantine

In inventaire des Hétéroptères aquatiques du Liban, basé sur des collections récentes et sur la littérature, est donné. Cet inventaire est accompagné de notes faunistiques et biogeographiques sur la base d'une comparaison avec la faune des pays avoisinants.

Aouatic Heteroptera of Lebanon. I. Faunistic Inventory.

Kevwords : Aquatic Heteroptera, Lebanon, Levantine region.

A list of the Lebanese aquatic Heteroptera, based on recent collections and litterature is given. A table of species found in neighbouring countries is presented together with synonymic, faunistic and zoogeographic notes.

\section{1. - Introduction}

Les données sur les Hétéroptères aquatiques de la vartie orientale de la Méditerranée sont beaucoup plus pauvres que celles de la partie occidentale. Ejles sont limitées, le plus souvent, à des listes fragmentaires à rechercher dans la littérature. Pour cette raison, nous avons jugé utile de publier ce travail qui représente, en quelque sorte, une contributinn à la connaissance non seulement des espèces actuellement recensées du Liban mais aussi des pays avoisinants.

1. Rijksuniversiteit Utrecht, Laboratorium voor zoölogische verologie en taxonomie, Plompetorengracht 9-11,3512 CA Utrecht, Nederland.

7. Laboratoire d'Hydrobiologie, U.A. 695 C.N.R.S., Université P. Sabatier, 118, route de Narbonne, 31062 Toulouse Cedex, France.

\section{2. - Matériel et sites étudiés}

Le matériel examiné provient du bassin versant de trois rivières libanaises : l'Oronte (= Assi) et le Litani dans la province intérieure ou Békaa et le Beyrouth dans la province côtière.

Les périodes de récoltes s'étalent sur trois années : juillet, août, septembre 1980 ; septembre, octobre 1981 ; mars, avril, mai 1982.

Treize stations ont été prospectées dont onze se situent sur les deux rivières de la Békaa : Oronte (stations 1 à 6), Litani (stations 7 à 11) et deux seulement sur le Beyrouth (stations 12 et 13).

\section{1. - Bassin de l'Oronte}

Station 1: Oronte-Hermel, alt. 650-600 m, hyporhithral 1a avec méandres et bras morts (lb), $\mathrm{T}^{\circ} \min 10-\max 15^{\circ} \mathrm{C}$; 
Station 2: Elain, alt $950 \mathrm{~m}$, ruisselet de source, $\mathrm{T}^{\circ} 9.12^{\circ} \mathrm{C}$;

Station 3: Labwé, alt. $1000 \mathrm{~m}$, ruisselet de source, $\mathrm{T}^{\circ}$ 9-12 ${ }^{\circ} \mathrm{C}$;

Station 4: Yammouné, alt. $1400-1300 \mathrm{~m}$, crénal $4 \mathrm{a}$ et $4 \mathrm{~b}\left(\mathrm{~T}^{\circ} 8-10^{\circ} \mathrm{C}\right)$, rhithral $4 \mathrm{c}$ $\left(\mathrm{T}^{\circ} 6-12^{\circ} \mathrm{C}\right)$, mares et lac temporaire $4 \mathrm{~d}$ $\left(\mathrm{T}^{\circ} 8,5-16^{\circ} \mathrm{C}\right)$;

Station 5: Chlifa, alt. $1200-1100 \mathrm{~m}$, rhithral 5a avec mouilles et replat $5 \mathrm{~b}, \mathrm{~T}^{\circ} 8,5$ $13^{\circ} \mathrm{C}$;

Station 6: Baalbek, alt. 1 150-1 $100 \mathrm{~m}$, crénal 6a $\left(\mathrm{T}^{\circ} 9-12,5^{\circ} \mathrm{C}\right)$, hyporhithral $6 \mathrm{~b}\left(\mathrm{~T}^{\circ} 9\right.$ $\left.16^{\circ} \mathrm{C}\right)$.

\section{2. - Bassin du Litani}

Stations 7 et 8 : Yahfoufa et Janta situées sur l'affluent Yahfoufa ; 7 , métarhithral, alt. $1200 \mathrm{~m} ; 8 \mathrm{a}$, hyporhithral, alt. $1100 \mathrm{~m}$, $8 \mathrm{~b}$, mare. $\mathrm{T}^{\circ} 11-14^{\circ} \mathrm{C}$;

Station 9: Anjar-Chamsine, alt. $1000-900 \mathrm{~m}$, affluent Ghozayel ; crenal $9 \mathrm{a}\left(\mathrm{T}^{\circ} 14\right.$ $18^{\circ} \mathrm{C}$ ), épipotamal supérieur $9 \mathrm{~b}$ $\left(\mathrm{T}^{\circ} 13 \cdot 20^{\circ} \mathrm{C}\right)$;

Station 10 : Ammik, alt. $850 \mathrm{~m}$, crénal 10a ( $\mathrm{T}^{\circ} 14$ $\left.16^{\circ} \mathrm{C}\right)$, marais $10 \mathrm{~b}\left(\mathrm{~T}^{\circ} 8.25^{\circ} \mathrm{C}\right)$;

Station 11 : Jib-Jennine, alt. $800 \mathrm{~m}$, le Litani, épipotamal inférieur pollué $\left(\mathrm{T}^{\circ} 12-23^{\circ} \mathrm{C}\right)$.

\section{3. - Bassin du Beyrouth}

Stations 12 et 13: Baalechmay-Hammana, alt. $1000-700 \mathrm{~m} ; 12=\operatorname{crénal}\left(\mathrm{T}^{\circ} 10-15^{\circ} \mathrm{C}\right)$, 13 = hyporhithral $\left(\mathrm{T}^{\circ} 8-27^{\circ} \mathrm{C}\right)$.

\section{3. - Liste commentée des espèces}

Quinze espèces ont été recensées dans les trois rivières prospectées. Nous donnons, ci-dessous, leur liste ainsi qu'un aperçu de leur distribution géographique et de leur écologie.

* Hydrometra stagnorum (Linnaeus) 1758 (= H. eromobia Kirtischenko).

Matériel : Baalbek, S6a, 20.10.81. 1 o ; Hammana, S1 3, 26.5.82, $7 \circ$ et $9 \%$ aptères, 2 \% macroptères. Distribution : Ouest-paléarctique, commune dans toute la sous-région méditerranéenne et connue de
l'Asie occidentale et centrale (Hoberlandt 1948; Kanyukova 1973 ; Nieser \& Montes 1984); signalée du Liban à Broumana et à Bcharré par Horváth (1926, 1929) et dans le Damour et l'Awali par Dia (1983).

Ecologie: vit en bordure des eaux stagnantes et courantes.

* Hebrus pusillus (Fall.) 1807.

Mat.: Hammana, \$13, 26.5.82, 1 \%.

Distr. : largement répandue dans la région paléarctique ; signalée du Liban à Bcharré (alt. $2050 \mathrm{~m}$ ), par Horváth (1929).

Ecol. : fréquente les milieux macrophytiques des eaux stagnantes ou peu courantes.

* Microvelia pygmaea (Dufour), 1833.

Mat. : Hermel, S1b, 17.5.82, 1 o. 2 \% : Janta, S8b, 24.5.82, $1 \%, 3$ \%.

Distr. : paléarctique, commune dans la sous-région méditerranéenne, trouvée en Asie jusqu'au Kamchatka mais les identifications des spécimens de l'Asie de l'Est ne sont pas toutes sûres (Hoberlandt 1948, Nieser 1978).

Ecol. : vit à la surface de l'eau, ordinairement parmi la végétation émergente, en courant très lent ou en bordure des eaux stagnantes.

* Rhagovelia nigricans (Burm.).

Mat. : Elaïn, S2, 25.9.81, $1 \sigma$ aptère.

Distr. : Afrique, Moyen-Orient et certaines îles méditerranéennes: Rhôdes et Chypre, (Horváth, 1913, 1926 ; Bodenheimer 1935 ; Hoberlandt 1951, 1952 ; Poisson 1957, Linnavuori 1960, Thiery 1981).

Ecol. : habite la zone rivulaire de ruisseaux.

* Velia affinis affinis Kolenati, 1853.

Mat.: Labwé, S3, 11.5.82, 1 ơ : Hammana, S13, 26.5.82, $4 \circ, 3 \&$, tous aptères.

Distr. : la sous-espèce typique de $V$. affinis est diffusée en Asie Mineure et dans la bordure orientale du bassin méditerranéen jusqu'en Iran et le Turqueménistan (Taminini 1949).

Ecol. : vit en bordure des eaux courantes.

* Gerris argentatus Schummel, 1832.

Mat.: Ammik, S10, 10.9.1981, 1 o macroptère.

Distr. : paléarctique, connue de Syrie (Horváth 1926) et de toute la sous-région méditerranéenne, mais peu commune. Au Japon et à Mandshurie, il s'agit de la sous-espèce $G$. argentatus babai Myriamoto (Nieser 1978).

Ecol. : ce Gerris se rencontre en bordure des eaux stagnantes, souvent parmi la végétation émergente. 
* Gerris costai fieberi Stichel, 1938.

Mat.: Yammouné, S4b, 11.9.80, $5 \circ, 8 \%, 2 \mathrm{Lv}$ IIJ/IV ; Janta, S8b, 24.5.82, I $\sigma$, I Lv V ; Anjar, S9b, 5.4.82, $2 \circ, 3$ \% ; Ammik, SLOb, 16.5.82, $1 \circ, 1 \%$; Hammana, $\$ 13,26.5 .82,2 \circ, 2 \%$; tous macroptères.

Distr.: la sous-espèce fieberi est connue d'Asie Mineure et de la région Est-méditerranéenne ; citée de Syrie par Wagner \& Zimmermann (1955).

Erol. : eaux stagnantes et courantes (Hoberlandt 1948) ; au Liban, cette sous-espèce peut atteindre des altitudes équivalentes à celles des formes d'Europe centrale et occidentale.

* Gerris thoracicus schummel, 1832.

Mat. : Baalbek, S6a, 24.5.82, 1 o , 1 \& : Janta, S8b, 24.5.82, $1 \sigma$; Anjar, S9b, 5.4.82, $2 \sigma, 3 \%$; Ammik, S10b, 16.5.82, 1 o. 1 \&; Jib-Jennine, S11, 16.5.82, $2 \sigma, 2$ \%; tous macroptères.

Distr. : paléarctique, très commune dans la sousréogion méditerranéenne.

Ecol. : eaux stagnantes et courantes.

* Gerris (Aquarius) ventralis (Fieber), 1861.

Mat. : Hermel, S1b, 17.5.82, $3 \sigma^{\circ}, 2$ apt.; Hammana, $S 13,26.5 .82,2 \circ, 2 \circ$; aptères.

Distr. : bordure orientale de la méditerranée où elle semble assez commune (Hoberlandt 1948).

Erol. : espèce fluviatile, écologie proche de celle de G. najas d'Europe centrale et occidentale.

* Corixa affinis Leach, 1818.

Mat.: Hermel, S1b, 25.9.81, 10, 2 \% ; Yammouné, S4. 11.9.80, 2 o, 1 \&, 14.10.81, 1 \% ; Ammik, S10b, 16.5.82, $2 \sigma, 5 \propto$; Jib-Jennine, $\$ 11,16.5 .82,1 \sigma$, 2 Q.

Distr. : largement répandue dans la région paléarctinue jusqu'en Inde, elle est très commune dans la sous-région méditerranéenne; connue de Yammouné (Horváth 1926).

Erol. : fréquente les eaux douces et saumâtres des milieux stagnants ou à courant très lent.

* Hesperocorixa parallela (Fieber), 1861.

Mat. : Yammouné, S4, 11.9.80, $1 \%$.

Distr. : sudest de l'Europe et de l'Asie Mineure ; connue de Syrie (Seidenstücker 1959).

Erol. : vit en eau stagnante ou à courant faible.

* Sigara albiventris (Horváth), 1911.

Mat. : Hermel, S1, 17.5.82, $1 \%, 1$ \%; Ammik, S1Ob, $8.8 .80,1 \sigma^{\circ}, 1$ \& $10.9 .80,2 \sigma^{\circ} ; 16.5 .82,5 \circ^{*}, 7$ \&, $2 \mathrm{Lv}$ IW/IV; Jib-Jennine, S11, 18.9.80, 3 \&.
Distr. : sud-ouest de l'Asie Mineure; décrite de Syrie par Horváth (1911, 1926).

Ecol. : préfère surtout les eaux stagnantes (mares, marais...) mais fréquente aussi les sections de rivières peu courantes (potamal).

* Sigara sarnani Hoberlandt, 1948.

Mat. : Ammik, S10, 16.5.82, $1 \propto$.

Distr. : nord de la province levantine.

Ecol. : espèce des eaux stagnantes.

* Notonecta maculata Fabricius, 1794

Mat. : Yammouné, S4d, 14.10.81, $1 \sigma, \mathrm{S} 4 \mathrm{c}, 23.5 .82$. 10,3 \&: Janta, S8, 24.5.82, 1 \&; Ammik, S10b, 10.5.82, $1 \mathrm{Lv}$ V ; Hammana, S13, 26.5.82, I \&, $6 \mathrm{Lv}$ I/III

Distr.: connue du sud-ouest de la région paléarctique et de quelques localités du sud-ouest de l'Asie jusqu'au Baloutchistan (Hoberlandt 1948); espèce commune dans toute la sous-région méditerranéenne.

Ecol. : fréquente les milieux stagnants ou à courant très lent pourvus d'un support solide (faciès pierreux, berges bétonées, ...) auquel les femelles attachent leurs ceufs.

* Notonecta viridis mediterranea Hutchinson, 1928 (=N. marmorea Esaki).

Mat. : Yammouné, S4d, 11.9.80, 2 Q, S4b, 14.10.81, 5 \&, S4d, 16.4.82, 3 \%.

Distr. : sud-est de l'Europe et sud-ouest de l'Asie, commune dans la partie orientale du bassin méditerranéen et en Asie Mineure (Hoberlandt 1948 ; Nie. ser 1978).

Ecol. : se rencontre en eau douce ou saumâtre mais surtout dans des faciès lentiques ou à courant faible.

\section{4. - Commentaire faunistique et biogéographique}

Sur le Tableau I nous avons représenté une distribution des Hétéroptères aquatiques et semiaquatiques connus au Liban et des pays avoisinants en excluant les espèces endémiques citées des régions limitrophes orientale et éthiopienne comme le delta de l'Euphrate et la haute Egypte.

La lecture de ce tableau nous permet de dégager un certain nombre de remarques faunistiques et biogéographiques : 
Tableau I : Distribution des Hétéroptères aquatiques et semi-aquatiques connus du Liban et des pays avoisinants : Chypre $(n=16)$; Turquie $(n=36)$; Irak $(n=20)$; Syrie $(n=23) ;$ Liban $(n=18) ;$ Israël $(n=43)$; Egypte $(n=26)$.

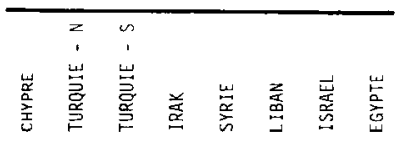

\begin{tabular}{|c|c|c|c|c|c|c|c|c|}
\hline Cymatia rogenhoferi (Fieb.) & & + & & + & & & & \\
\hline Corixa abfinis Lch. & + & + & + & + & + & + & + & + \\
\hline C. jakovleffi Hirv. & & & + & + & & & + & \\
\hline C. punctata (Iel.) & & + & + & & & & + & \\
\hline Helioconisa vermiculata (Put.) & & & & + & & & & + \\
\hline Hesperocorixa parallela (Fb.) & & & + & & + & + & & \\
\hline H. occulta (Lbl.) & & + & & + & + & & & \\
\hline Sigara albiventris [Hu. I & & & + & + & + & + & & \\
\hline S. assimilis |Fb.| & & + & + & + & & & & \\
\hline S. emesa Sdst. & & & & & + & & & \\
\hline S. hoggarica (Pois.] & & & + & & & & & + \\
\hline S. Lateralis (Lch.) & + & + & + & + & + & + & + & + \\
\hline S. mayri (Fb.) & & & + & & & & & + \\
\hline S. nigholineata (Fb.) & + & + & + & & + & + & + & \\
\hline S. samani $(H b)$. & & & + & & + & + & & \\
\hline S. selecta [Fb.] & + & & & & & & & + \\
\hline S. sexlineata (Rt.) & & & & & & & + & + \\
\hline S. striata (L.) & & & & & + & & + & \\
\hline Micronecta isis Horv. & & & & & & & + & + \\
\hline M. perparva Horv. & & & & & & & + & \\
\hline M. plicata $(C)$, & & & & & & & + & + \\
\hline H. scutellaris & & & & & & & + & + \\
\hline ochterus marginatus (Lat.) & + & + & + & & + & & & \\
\hline 0 . strigicollis Hu. & & & & & & & + & \\
\hline Aphelocheirus aestivalis $|F|$. & & & + & & & & + ? & \\
\hline Ilyocoris cimicoides $(1)$. & & + & + & & & & + & \\
\hline $\begin{array}{l}\text { Naucoris maculatus } F \text {. } \\
\text { Heleocoris minuscula (wk.) }\end{array}$ & & & + & & & & $\begin{array}{l}+ \\
+\end{array}$ & \\
\hline Lethocerus fakir Gs. & & & & & & & + & + \\
\hline L. patruelis $(S t a l \mid$ & & & + & + & & & & \\
\hline Limnogeton fieberi $M$. & & & & & & & + & + \\
\hline Diplonychus wrinator 1 D́.l & & & & & & & + & + \\
\hline Nepa cinerea 1. & & + & + & & & + & + & + \\
\hline Laccotrephes fabricii stal & & & & & & & + & + \\
\hline Ranatra Rinearis (L.) & & + & + & & & & + & \\
\hline R. vicina $\mathrm{Sg}$. & & & & & & & + & + \\
\hline Plea minutissima Lch. & + & + & + & + & + & & + & \\
\hline P. Letourneuxi Sg. & & & & & & & + & + \\
\hline Notonecta maculata $F$. & + & + & & & & + & + & + \\
\hline N. viridis mediterranea Htch. & & & & + & $+?$ & + & + & + \\
\hline Anisops sardea (H.-S.) & + & & + & + & & & + & + \\
\hline Mesovelia vittigera $\mathrm{Hv}$. & & & + & + & + & & + & + \\
\hline Hydrometra stagnoram $[L]$. & + & + & + & + & + & + & + & + \\
\hline Hebrus pusillus (Fal.) & & + & + & & + & + & + & + \\
\hline H. syriacus Ho. & + & & & & + & & + & \\
\hline Microvelia pygmaea $10 u$ f. & + & & + & & & + & + & + \\
\hline Rhagovelia nigricans (Burm.] & + & & & & + & + & + & + \\
\hline Velia abfinis affinis Kol. & + & + & + & + & + & + & + & \\
\hline Gerris argentatus Sch. & + & & + & + & + & + & + & \\
\hline G. asper Fb. & & + & & & + & & + & \\
\hline G. costai fieberi st. & & + & + & + & + & + & + & \\
\hline G. gibbifer Sch. & & + & + & & & & + & \\
\hline G. lacustris (1.) & & + & + & + & & & & \\
\hline G. paludum $|F|$. & & + & + & + & + & & it & \\
\hline G, thoracicus Sch. & + & + & + & + & + & + & + & \\
\hline G. ventratis $\left\{F_{6}.\right\}$ & + & & + & & & + & + & \\
\hline Naboandelus bergevini $\mathrm{Bg}$. & & & & & & & + & + \\
\hline
\end{tabular}


- Mesovelia furcata citée par Bodenheimer (1937) correspond, ici, à $M$. vittigera;

- certaines citations de Veliidae, comme Velia cur. rens (F.) et $V$. rivulortum $(F$.), sont à revoir car elles ne coïncident pas avec leur distribution géographique; Velia nervosa est connue de Turquie ;

- Gerris paludum bergrothi (Lbl.) (=Cylindrostethus bergrothi Lbl.) et G. palmonni Wgn. ne sont pas assez différentes pour être distinguées comme des sous-espèces et se considèrent comme synonymes de G. paludum (F.) (Kanyukova 1982):

- les citations de Gerris najas cinereus Put de Palestine par Jaczewski (1934), G. najas (De $\dot{\mathrm{G}}$.) par Bodenheimer (1937) demandent confirmation; il s'agirait probablement de $G$. ventralis (Fb.), G. cinereus présente plutôt une distribution Ouest-méditerranéenne ;

- Hydrometra aegyptia Hf. \& Ev. est une espèce endémique de l'Egypte; Microvelia hozari $\mathrm{Hb}$. est endémique de la Turquie, seulement la sousespèce $M$.h. macani Brown est connue de l'Irak: $M$. gracillima est connue d'Israël; $\boldsymbol{M}$. priesneri $\mathrm{Hb}$., M. waelbrocki $\mathrm{Hk}$, et Gerris brevirostris $\mathrm{Bg}$. sont connues d'Egypte :

- Aphelocheirus aestivalis (F.) est connue de Turquie (Mersin); elle est citée d'Egypte par Jaczewski (1936) :

- Lethocerus cordofanus Mayr 1853, est synonyme de L. fakir (Gistel 1848). L. pruinosus Dufour 1863 et $L$. niloticus var. persicus Mont. 1898 sont des synonymes de L. patruelis (Stäl 1858) (Kanyukova \& Kerzhener 1980); l'identité du Lethocerus de Palestine (L. patruelis, Bodenheimer 1937), est douteuse :

- la citation de Nepa sardinensis Hf. d'Israël par linnavuori (1960) est, selon Tamanini (1973), une erreur ; la Ranatra de l'Irak est $R$. unicolor Sc., espèce assez proche de $R$. vicina Sign. ; la citation de $R$. linearis de Palestine, par Bodenheimer (1937) demande confirmation :

- jusqu'à présent le genre Notonecta n'est représenté dans cette région orientale que par deux espèces : $N$. maculata F. et $N . v$. mediterranea Htch. et les mentions concernant d'autres espèces, comme $N$. glauca L. par exemple, nous semblent douteuses; cependant, nous considérons les citations de $N$. glauca (L.) d'Israël et de Syrie (Horváth 1913, 1926) et du Liban (Dia 1983) comme des N.v. mediterranea; toutefois, nous soulignons que les deux Notonectidae Anisops debilis perplexa Gerst. et Enithares rhodopis Htch., connues d'Egypte, ne figurent pas dans notre tableau;

- la plupart des citations de Micronecta de l'EstMéditerranéen demandent confirmation. $M$. parvula Lb. correspond à $M$. perparva (Linnavuori 1960) ;

-- Cymatia bonsdorffi (Sahlb.), Callicorixa concinna (Fieb.) et Hesperocorixa linnei (Fb.) se rencontrent en Turquie et Sigara kervillei Poiss, est endémique de ce pays; la citation de Arctocorixa carinata (Sahlb.) et de Sigara striata (L.) de Palestine par Bodenheimer (1937) est douteuse et demande confirmation ; Sigara hedenborgi Lbl. se rencontre en Egypte; Sigara emesa Sdst. est connue seulement de Syrie (Seidenstücker 1959).

\section{5. - Conclusion}

Vingt espèces d'Hétéroptères aquatiques sont actuellement connues du Liban, auxquelles se rajouteraient probablement $M$. vittigera, $H$. syriacus, $P$. minutissima et $\boldsymbol{A}$. sardea (Tableau I).

Dans ce peuplement nous distinguons principalement deux groupes d'espèces:

- le premier groupe se compose de dix especes lar. gement répandues dans la région paléarctique ou euro-sibérienne (région méditerranéenne com. prise). Ce sont : $H$. stagnonum, $H$. pusillus, $G$. argentatus, $G$. thoracicts, $N$. cinerea, $N$. maculata, C. affinis, $S$. lateralis, $S$. nigrolineata, et probablement $M$. pygmaea;

- le second groupe comporte six espèces ou sousespèces qui sont considérées comme des éléments caracteristiques de la région Estméditerranéenne; il s'agit de $V$. affinis affinis, $G$. costai fieberi, $G$. ventralis, N.v. mediterranea, $H$. parallela et $S$. samani auxquelles nous ajoutons éventuellement $S$. albiventris qui est un élément confiné au sud-ouest de l'Asie.

D'une manière générale le peuplement de la partie orientale du bassin méditerranéen ne se trouve individualisé qu'au nord et au sud de la province levantine. Ainsi, nous distinguons deux noyaux d'espèces : 
- au nord et au nord-est du Levant : Cymatia rogenhoferi, Corixa jakovletfi, Sigara assimilis, Gerris lacustris et Lethocerus patruelis;

- au sud du Levant : Sigara sexlineata, Limnogeton fieberi, Diplonychus urinator, Laccotrephes fabricii, Ranatra vicina, Plea letourneuxi, Naboande. lus bergevini et Lethocerus fakir.

Soulignons, en particulier, dans le deuxième novau d'espèce, la présence de trois éléments éthiopirns, $L$. fieberi, D. urinator et $L$. fabricii, pour lesquels le bassin du Jourdain (sud du Levant) constitue la limite nordest de leur aire de répartition.

\section{Travaux cités}

Bodenheimer (F.S.). 1937. - Prodomus Faunae Palestinae. Mem, Inst, XXXIII, 286 p., 4 figs.

Brown (E.S.). 1953. - Notes on aquatic Hemiptera from Syria and Iraq. Ann. Mag. Nat. Hist. Ser., 12, 6: 579-600.

China (W.E.). 1938. - Hemiptera from Iraq, Iran and Arabia. Zool. Ser. Field Mus. Nat. Hist. 20 (8) : 427.437.

Dia (A.). 1983. - Recherches sur l'écologie et la biogéographie des cours d'eau du Liban méridional. Thèse d'Etat, Univ, Aix-Marseille III, 302 p.

Hoberlandt (L.). 1948. - Results of the zoological scientific expedition to Turkey. Aquatic and Semiaquatic Heteroptera of Turkey. Acta Ent. Mus. Pragae, 26: 1-74. $17 \mathrm{pls}$.

Hoberlandt (L.). 1952. - On some Hemiptera-Heteroptera of Cyprus. Ibid. $28: 109-116$.

Horváth (G.). 1911. - Hemiptera nova vel minus cognita e Regione Palaearctica. Ann. Mus. Nat. Hung. 9: 573-610.

Horvath (G.). 1913. - Aquatic and semiaquatic Rhynchoata from the Lake of Tiberias and its immediate vicinity. J. As. Soc. Beng. IX (5): $477-480$.

Horváth (G.). 1926. - Hémiptères. In Voyage zoologique d'Henri Gadeau de Kerville en Syrie : 107-119. Tome I, 365 p. Imp. Lecerf fils, Rouen.

Horváth (G.). 1929. - Rhynchoten aus Palästina und Syrien. Sitzber. Akad. Wiss. Wien (Naturw. I), 138: 330.332.
Jaczewski (T.), 1934, - Notes on some palearctic aquatic and semi-aquatic Heteroptera, chiefly from S-E Europe. Ann. Mus. Zool. Pol, 10: 267-287. pl. 46, 47.

Jaczewski (T.). 1936. - Contributions to the knovledge of aquatic Heteroptera of Egypt. Ann. Mus, Zool Pol, 11 : 171-211, pl. 27, 28.

Jaczewski (T.). 1962. - Redescription of Corixa jakowleffi (Horv.). Bull. Acad. Pol. Sc. (Cl. II) 10:325-329.

Jaczewski (T.), 1964. - Notes on some aquatic and semiaquatic Heteroptera from Iraq. Ibid. $12: 263.268$.

Kanyukova (E.V.) 1973. - O rasprostranenii Hydrometridae (Heteroptera) fauny SSSR. Zool. Zhum. 52: 1253-1254.

Kanyukova (E.V.). 1982. - Vodomerki (Heteroptera, Gerridae) fauny SSSR. Trudy Zool. Inst. Akad. Nauk. SSSR. 105: 62.93 .

Kanyukova (E.V.) \& Kerzhner (I.M.). 1980. - K sinonimike i rasprostraneniyu poluzhestkokrylych roda Lethoce. rus Mayr (Heteroptera, Belostomatida starogo sveta. Ent. Obozr. 59 : $597-599$.

Lindberg (H.). 1948. - On the insect fauna of Cyprus I, II. Hemiptera-Heteroptera. Comment. Biol. 10 (7) : 1-175.

Linnavuori (R.). 1960. - Hemiptera of Israel. Ann. Zool. Soc, Vanamo 22 (1) : $1-71$.

Linnavuori (R.). 1964. - Herniptera of Egypt, with remarks on some species of the adjacent eremian region. Ann. Zool. Fenn., 1 : 306-356.

Nieser (N.). 1978. - Heteroptera : 280-285. In Limnofauna Europaea (J. Illies ed.). G. Fischer Verlag. Stuttgart, Swets \& Zeit. Amsterdann.

Nieser (N.) \& Montes (C.). 1984. - Lista faunistica y bibliográfica de los Heterópteros acuáticos (Nepomorpha y Gerromorpha) de la peninsula Ibérica. As. Esp. Limnol., 73 p.

Poisson (R.). 1957. - Hemiptera, Heteroptera, Hydrocorisae et Geocorisa-Gerroidea. South African Animal Life, 4 (8) : 327-373.

Seidenstüicker (G.). 1959. - Sigara emesa n. sp. und einige Corixiden aus der Türkei und Syrien. Rev. Fac. Sc. Univ. Istanbul (B) 24 : 33-38.

Tamanini (L.). 1949. - 3c Contributo allo studio del genere Velia Latr. (Hem. Het: Veliidae), Acta Ent. Mus. Nat. Pragae 26 (366) : 1.10.

Wagner (E.) \& Zimmermann (S.). 1955. - Beitrag zur Systematik der Gattung Gerris F. (Hemiptera-Heteroptera, Gerridae). Zool. Anz. 155 : 177-190. 